\title{
Design and Performance of a High Resolution Electrospray Ion Source for a Magnetic Sector Mass Spectrometer with a Heated Capillary Inlet
}

\author{
Longfei Jiang and Mehdi Moini. \\ Department of Chemistry and Biochemistry, University of Texas at Austin, Austin, Texas, USA
}

\begin{abstract}
The design and performance of an electrospray ion source for a high resolution magnetic sector mass spectrometer that utilizes a heated capillary has been presented. Low pressure, high sensitivity, stable electrospray, low flow rates, and low electronic noise were important factors in achieving high resolution electrospray mass spectrometry. A unit mass resolution has been achieved for biomolecules with $\mathrm{MW}>12,000$, and an accuracy of $1.4 \mathrm{ppm}$ has been achieved for the average molecular weight of bovine insulin. (J Am Soc Mass Spectrom 1995, 6, 1256-1261)
\end{abstract}

E lectrospray ionization (ESI) has become a successful mass spectrometric technique for analysis of large biological molecules [1-3]. Currently, ESI has been adapted to a variety of mass spectrometers, which include quadrupole, time-of-flight, ion cyclotron resonance, quadrupole ion trap, and magnetic sector instruments [4-9].

Although several electrospray source designs have been published for low resolution study [6-14], source designs for sector instruments that can achieve high resolution (unit mass resolution for $M W>10,000$ ) are limited $[15,16]$. There are several advantages of the use of high resolution ESI (resolution > 10,000), which include accurate identification of the charge state of multiply charged ions by simply counting the number of peaks in a unit mass interval and accurate mass measurement of high molecular weight multiply charged ions. Chapman et al. [10] recently demonstrated that even a modest gain in resolution (2400-3000) significantly increases the precision of the experimental determination of molecular mass, particularly at higher masses.

Two primary desolvation techniques that are applicable to ESI are (1) counterflow of heated bath gas [11] and (2) heated capillary tube [12]. In the counterflow of heated bath gas, which is currently the most commonly used ESI desolvation technique, a countercurrent of heated gas that flows against solvated ions formed at atmospheric pressure aids the desolvation process of the electrospray-produced charged droplets.

Address reprint requests to Dr. Mehdi Moini, Department of Chemistry and Biochemistry, University of Texas at Austin, Austin, TX 78712 .
Chait and co-workers [12] have described the use of a heated capillary tube (HCT) as a convenient and effective desolvation method that does not require a countercurrent of heated gas. Desolvation with this system is effected by the use of controlled heat transfer through a long capillary tube and collisional activation in the region of reduced pressure between the capillary tube exit and the skimmer.

Recently, Dobberstein and Schroeder [15] adapted an atmospheric pressure ionization source, which was originally designed for a quadrupole mass spectrometer, to a Finnigan-MAT (San Jose, CA) double-focusing mass spectrometer. Their source, which utilized a heated capillary tube, can achieve a resolution of 20,000 and a mass deviation of $1 \mathrm{ppm}$ for the $13+$ ion of myoglobin, MW 16,951. However, (1) no details with regard to the construction of the source were given, (2) it is offered only for the Finnigan-MAT magnetic sector mass spectrometers, and (3) it uses an expensive and complicated octapole lens system and additional power supplies.

To date, no detailed source design that achieves high resolution $(>10,000)$ for a sector instrument has been published. Previously, we successfully converted a Finnigan-MAT TSQ-70 thermospray ionization interface to an electrospray interface by using a heated capillary tube [13]. This source, however, was capable of achieving only a unit mass resolution for MW < 1000. In this article, we report on the detailed design of an ESI interface to our VG ZAB high resolution double-sector mass spectrometer which (1) is capable of achieving a unit mass resolution for compounds with MW $>12,000$, (2) does not use additional high voltage power supplies (i.e., all high voltage supplies are from 
the ZAB mass spectrometer), (3) requires only two additional mechanical pumps, and (4) distances between the HCT and the first skimmer and between the first and second skimmers are adjustable to provide maximum sensitivity in the low resolution mode and high resolutions in the high resolution mode.

\section{Experimental}

Sample solutions were introduced via infusion into the electrospray source with a Harvard Apparatus (South Natick, MA) Model 22 syringe pump at a flow rate of $1-5 \mu \mathrm{L} / \mathrm{min}$. The syringe used for infusion was connected to the electrospray needle via Teflon tubing. Samples were purchased from Sigma Chemical Company (St. Louis, MO) and were used without further purification. All samples were dissolved in a 47:47:6 $(v / v)$ solution of water + methanol + acetic acid. Sample concentrations were $44 \mathrm{pmol} / \mu \mathrm{L}$ for gramicidin $\mathrm{S}, 9 \mathrm{pmol} / \mu \mathrm{L}$ for bovine insulin, and $50 \mathrm{pmol} / \mu \mathrm{L}$ for cytochrome $c$.

A VG ZAB mass spectrometer (Fisons Instruments, Manchester, UK) with an OPUS data system was used to acquire and process data. Full scan mass spectra were acquired at approximately 1000 resolution (10\% valley) by scanning the magnetic field over the desired mass range. High resolution mass measurements were made by accelerating voltage scans over a narrow mass range. All mass spectra were acquired in profile mode and several scans were averaged to improve the signal-to-noise ratios.

\section{Design}

The design of the electrospray ion source for a VG $\mathrm{ZAB}$ mass spectrometer is shown in Figure 1. The analytes were electrosprayed through the $0.12-\mathrm{mm}$-i.d. dome-tipped needle (J) (SGE Inc., Austin, TX) by keeping the needle at a potential higher (for positive ions) than that of the inlet heated capillary $(\mathrm{H})$. The analyte flow rate was kept constant by a syringe pump. The highly charged droplets were carried into the heated capillary tube $(\mathrm{H})$ by the flow of gas from atmospheric pressure to vacuum and by the potential difference between the needle and the capillary tube. Then the charged droplets migrated along the 11- $\mathrm{cm}$-long, 1.5$\mathrm{mm}$-o.d., 0.5-mm-i.d. (glass-lined interior) heated capillary tube (or HCT, part H; SGE Inc.) and entered the ESI source.

The capillary tube $(\mathrm{H})$ was wrapped with heater tape to maintain a temperature of about $200^{\circ} \mathrm{C}$ on the tube wall as measured by an attached thermocouple. Droplets that entered the HCT were strongly solvated, but as they traveled toward the mass spectrometer the temperature inside the tube facilitated evaporation of the solvent so that ions that exited the capillary tube were almost completely desolvated. Further desolvation was effected by collisional activation in the region of reduced pressure between the capillary and the first skimmer (E). The second skimmer (Q), which was secured by screws on the bottom plate of the lens assembly, and efficient pumping of the area between the first and second skimmers were necessary to obtain pressures low enough for high resolution studies. Skimmers 1 and 2 were fabricated in-house and have angles of $90^{\circ}$ and $60^{\circ}$, respectively. The completely desolvated ions entered the lens assembly, which includes an extractor electrode (C), focus lenses (B), and a guard plate $(Y)$ with exit slit. Similar to the commercial fast-atom bombardment source of the ZAB mass spectrometer, there are two holes on the guard plate that are well fitted to the first collision gas cell assembly (A) of the ZAB mass spectrometer to ensure the alignment of the ESI source with the ion optic system of the instrument.

The dome-tipped needle was secured by a Swagelok (Arthur Valve \& Fitting, Austin, TX) union (N) on the stopper $(T)$ at the entrance end of the tube housing $(O)$. To suppress arcing, dry nitrogen may be introduced through a hole on the stopper. The tip of the needle was placed about $10-15 \mathrm{~mm}$ away from the entrance end of the HCT. The HCT was secured by a Swagelok

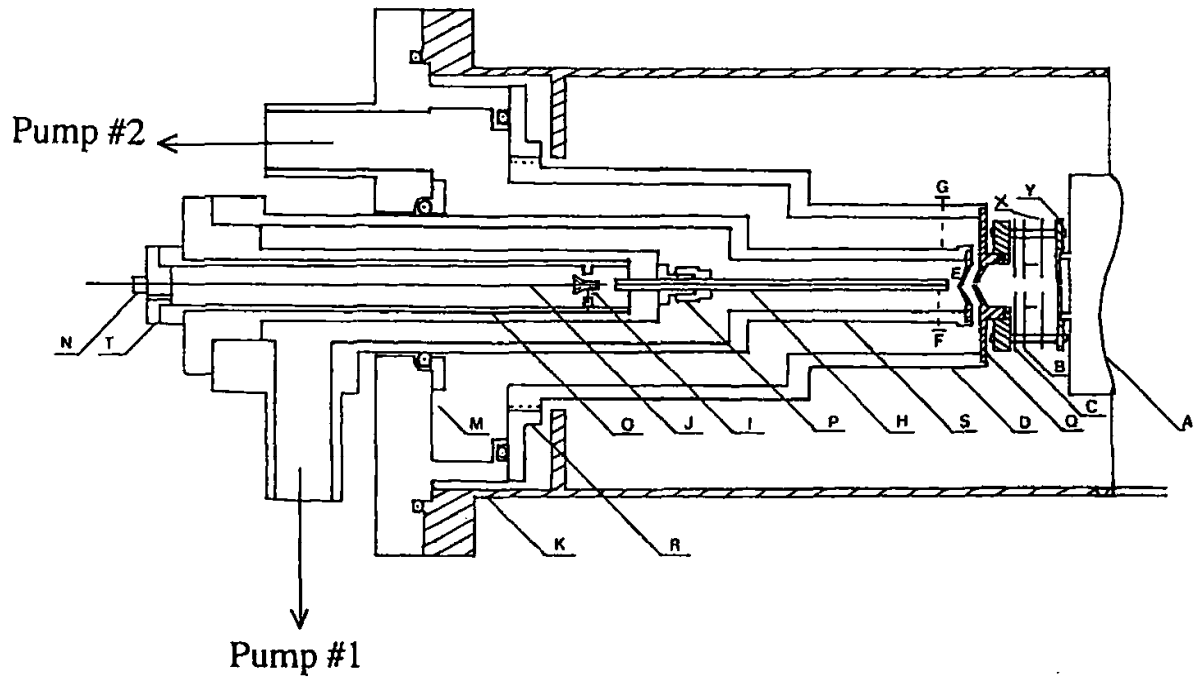

Figure 1. Schematic diagram of the ESI source on the ZAB double-focusing sector instrument (see text for details). A, first collision gas cell; $B$, focus lens $1 ; C$, extractor electrode; $D$, tube housing; $E$, first skimmer; $F$, set of screws; $G$, set of screws; $H$, heated capillary tube; I, set of screws; J, 0.12 -mm-i.d. dome-tipped needle; $M$, flange; $N$, Swagelok union; $O$, tube housing; $P$, Swagelok fitting; $Q$, second skimmer; $R$, round plate; $S$, tube housing; T, stopper; $X$, grounded lens; $Y$, guard plate (at ground potential). Focus lens 2 is located outside of the source vacuum housing; therefore, it is not shown in this figure. Dashed areas are composed of stainless steel. 
fitting $(P)$ on the other end of the tube housing $(O)$. The exit end of the HCT was $5 \mathrm{~mm}$ from the orifice of the first skimmer. This skimmer was fastened by two screws to the top of tube housing (S), which was held vacuum tight to the flange $(\mathrm{M})$ by an $\mathrm{O}$-ring. This arrangement allowed the distance between the first and second skimmers to be changed from the outside without breaking the vacuum by moving the tube housing (S). The optimal value for this distance is $2-3$ $\mathrm{mm}$. The lens assembly (including the second skimmer) was tightly fitted into the top end of the tube housing (D). The other end of $D$ was threaded to a round plate $(R)$ that was connected to the protruding edge of flange (M) by four nylon screws and sealed with an O-ring. This construction allows for minor adjustment of the tube housing (D) simply by rotating (M). Because the alignment pins were fixed to the first collision gas assembly, rotation of (D) will increase or decrease the length of this tube housing, so that the lens assembly can fit tightly onto the first collision gas cell assembly. The alignment of the dome-tipped needle, HCT, and first skimmer with the rest of the system was achieved by means of three sets of screws [(I), (F), and $(G)]$. Each set was comprised of three screws located around the tube housing at $120^{\circ}$ angles. A straight wire with a diameter of $0.4 \mathrm{~mm}$ was used to check the alignment by inserting it through the HCT, first skimmer, second skimmer, and exit slit.

The tube housing $(O),(S)$, and (D) divided the ESI source into three separated pumping stages that were supported by two independent rotary pumps (pump 1 is a DuoSeal 1397 operating at $400 \mathrm{rev} / \mathrm{min}$ with a free air displacement capacity of $500 \mathrm{~L} / \mathrm{min}$; pump 2 is a DuoSeal 1376 operating at $525 \mathrm{rev} / \mathrm{min}$ with a free air displacement capacity of $300 \mathrm{~L} / \mathrm{min}$; Welch Vacuum Technology Inc., Skokie, IL) and the source diffusion pump (Diffstak MK2, $700 \mathrm{~L} / \mathrm{s}$, Edwards, West Sussex, England). Typical pressures achieved at different stages during electrospray were as follows: first stage (including the space between the exit end of the HCT and the first skimmer), 5-10 torr; second stage (including the space between the two skimmers), $5 \times 10^{-2}$ torr; source housing, $2 \times 10^{-5}$ torr; analyzer, $1 \times 10^{-7}$ torr. Because the pressures of the first two stages fell within the arc zone, the two supporting rotary pumps had to be floated at the source high voltage to avoid arcing. This was accomplished by mounting the vacuum pumps on a piece of hardwood and isolating the pumps from the motor by using a Delrin pulley on the motor instead of the original metal pulley [5].

The typical values of the applied voltages were: dome-tipped electrospray needle, approximately $8 \mathrm{kV}$; $\mathrm{HCT}, 5.08 \mathrm{kV}$; first skimmer, $5.02 \mathrm{kV}$; extractor electrode, $4.98 \mathrm{kV}$; first focus lens, $3.8 \mathrm{kV}$; second focus lens (in first collision gas cell assembly), $3.3 \mathrm{kV}$. All high voltages were obtained from the high voltage power supplies of the $\mathrm{ZAB}$. The cesium ion gun power supply was used for the electrospray needle. For the voltage applied to the HCT, we used the collision cell voltage; for the voltages applied to the first and second skimmers, we used the ion repeller (plus a $30-\mathrm{V}$ battery in series with it) and the source voltage, respectively; for the voltage applied to the extractor, we used the filament voltage; for the voltages applied to the first and second focus lenses, we used the focus voltages provided by the source control unit of the mass spectrometer. Later, however, because of internal arcing of the source control unit under ESI conditions, we replaced the source control unit and collision cell voltage with a homemade, voltage divider. The source voltage was used as the input voltage of the voltage divider.

The entire electrospray source except for the needle, capillary, skimmers, and lens assembly was made of Delrin and mounted on a Delrin flange, which was substituted for the metal flange used for the ZAB mass spectrometer sources. This design dramatically reduced the possibility of arcing inside the source and between the source and its housing. Changing between the ESI source and other ZAB mass spectrometer sources was now accomplished more rapidly and with greater ease.

\section{Experimental Parameters to Achieve High Resolution}

High sensitivity, low pressures, stable electrospray, and stable electronics are important conditions to achieve high resolution on sector instruments.

High sensitivity of the heated capillary-based ESI source depends on several factors, which include the inner diameter and length of the capillary, the distance between the end of the capillary and the first skimmer, the distance between the two skimmers, and the source ion optics. To study the performance of the mass spectrometer interface, the $44-\mathrm{pmol} / \mu \mathrm{L}$ solution of gramicidin $S$ in a mixture of water + methanol + acetic acid $(47: 47: 6 \mathrm{v} / \mathrm{v})$ was infused into the electrospray needle. Either the signal intensity at the mass spectrometer detector or the ion current at the second skimmer was then used to study the effect of various factors.

Three HCT inner diameters were examined: $0.2,0.5$, and $0.75 \mathrm{~mm}$; the $0.5-\mathrm{mm}$ size was found to be optimal. Consistent with our previous experience [13], the $0.75-\mathrm{mm}$ tube produced pressure too high to achieve high resolution spectra, and the $0.2-\mathrm{mm}$-i.d. capillary reduced signal intensity beyond the range acceptable for high resolution study and it required frequent cleaning due to clogging.

During the initial stages of development, we used a 30-cm-long heated capillary tube. At this length the capillary was long enough to let us visually observe the electrospray process and the position of the ESI needle with respect to the entrance of the HCT. With a 30-cm-long heated capillary, however, the sensitivity was not high enough to achieve high resolution. The 
HCT length was therefore reduced to $11 \mathrm{~cm}$. - the minimum length possible with our interface. This length was used throughout the experiment. The distance between the end of the HCT and the first skimmer had an important effect on pressures in various stages of the vacuum system as well as on ion signal intensity. Table 1 summarizes this factor. Hence, a distance of $5 \mathrm{~mm}$ was used as a trade-off between maximum signal intensity and minimum system pressure. Although there is a considerable sensitivity gain at 6 and $7 \mathrm{~mm}$, the pressure in the accelerating region was too high to be able to resolve the isotope cluster of cytochrome $c$. However, this sensitivity gain was exploited in the low resolution mode. The distance between the two skimmers was adjusted from 1 to $7 \mathrm{~mm}$. At short distances (1-2 mm), the pressure in the source housing was too high for HRMS ( $>5 \times 10^{-5}$ torr). In addition, the signal intensity at this distance was below the optimum intensity, probably due to scattering. A distance of approximately $3 \mathrm{~mm}$ produced the optimum conditions, that is, source pressure below $2 \times 10^{-5}$ torr, and maximum signal intensity. After working under these conditions for a week, a slight burnt spot of approximately $1 \mathrm{~mm}$ in diameter was observed around the tip of the second skimmer that indicated that most ions passed through the 0.5 -mm-i.d. hole. Distances of more than $4 \mathrm{~mm}$ resulted in loss of sensitivity. Therefore, distances of 2.5-3.5 mm were used throughout the experiment.

Three skimmer hole sizes of $0.3,0.5$, and $1.0 \mathrm{~mm}$ for $\mathrm{S} 1$ and two skimmer hole sizes of 0.5 and $1.0 \mathrm{~mm}$, for S2 were examined. The $1.0-\mathrm{mm}$-i.d. skimmers on both S1 and S2 produced a source pressure of $>5 \times 10^{-5}$ torr. The use of $0.3-\mathrm{mm}$ i.d. on S1 resulted in loss of signal with respect to the optimum conditions, that is, $0.5-\mathrm{mm}$ i.d. for both $\mathrm{S} 1$ and S2. Therefore, $0.5-\mathrm{mm}-\mathrm{i} . \mathrm{d}$. skimmers were used.

The temperature of the heated capillary also was optimized for maximum sensitivity. The optimum HCT temperature depends on the length of the HCT. Higher temperatures were needed for the shorter capillaries. For the 11-cm capillary that was used in this study, the optimum temperature range was between 200 and $220^{\circ} \mathrm{C}$. Below $180^{\circ} \mathrm{C}$, the temperature was too low to promote complete desolvation, and the signal intensity was less than optimal. Above $230^{\circ} \mathrm{C}$, coloration and eventual deformation of the Delrin vacuum housing occurred. In the optimum temperature range, no noticeable change in performance was observed for different analytes. (See [13] for more information on the relationship between the temperature of the HCT and solvent composition, etc.).

After high sensitivity and low pressure were achieved, the stability of the electrospray process and the voltages of the lens elements under ESI condition are other important factors in achieving high resolution. The following tuning procedure was used to obtain high resolution in these experiments:

1. Gramicidin $\mathbf{S}$ was used as the tuning compound. This compound was used because the doubly charged ion of gramicidin $S(m / z 572)$ produces a very strong peak under ESI conditions, and one can observe the effect of varying the tuning parameters on the peak in real time (on the mass spectrometer scope).

2. The ion optics of the instrument were then adjusted to maximize the intensity of the doubly charged ion of gramicidin S. Because, of the difference in the kinetic energy spread of the singly charged ions and multiply charged ions, the accelerating voltage/electric sector voltage ratio needed to be varied. On the $Z A B$, this was done by adjusting a knob called ion energy that actually changes the accelerating voltage.

3. After the maximum signal intensity for the doubly charged ions of gramicidin S was achieved, the mass spectrometer was tuned to a resolution of approximately 10,000 by narrowing the source slit until the peak intensity was $\sim 10 \%$ of its original intensity and by narrowing the detector slit to obtain a triangular peak shape with an additional reduction in intensity of $\sim 50 \%$, and by adjustment of the lens voltages. This tuning procedure is the same as that suggested by the manufacturer of the $\mathrm{ZAB}$.

4. Under these conditions, the noise level on the monoisotopic peak of the doubly charged ion of

Table 1. The effect of the distance between the end of the heated capillary tube and the first skimmer on the source pressure and ion current at the second skimmer

\begin{tabular}{lccc}
\hline $\begin{array}{c}\text { Distance } \\
(\mathrm{mm})\end{array}$ & $\begin{array}{c}\text { Source pressure, } \\
\text { heater off } \\
\left(10^{-5} \text { torr }\right)\end{array}$ & $\begin{array}{c}\text { Source pressure, } \\
\text { heater on } \\
\left(10^{-5} \text { torr }\right)\end{array}$ & $\begin{array}{c}\text { lon current on } \\
\text { 2nd skimmer, } \\
\text { heater on } \\
(\text { nA })\end{array}$ \\
\hline \hline 1 & 6 & 8 & - \\
2 & 3 & 2 & 20 \\
3 & 2 & 1.7 & 20 \\
4 & 1.7 & 1.5 & 30 \\
5 & 1.5 & 1.5 & 60 \\
6 & 1.5 & 9 & 150 \\
7 & 3 & 10 & 200 \\
8 & 7 & 8 & 60 \\
\hline
\end{tabular}




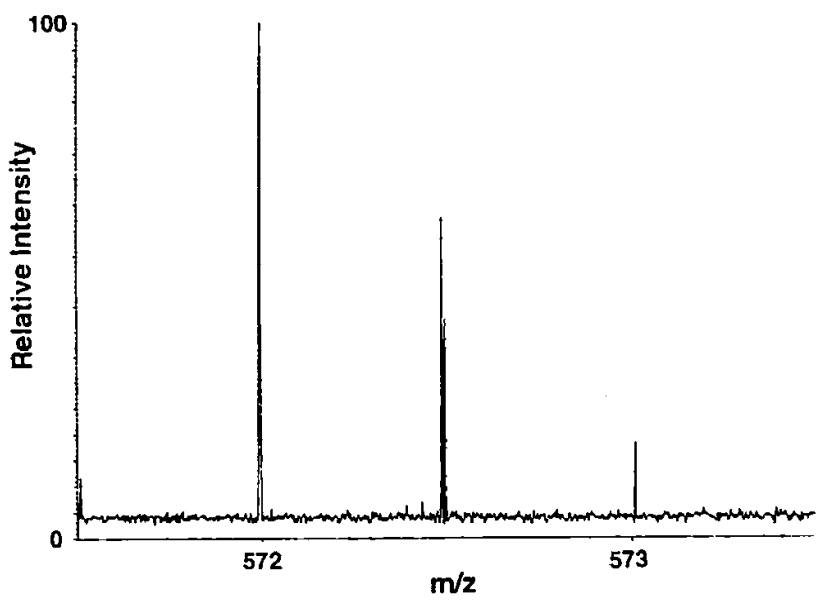

Figure 2. ESI spectrum of the doubly charged ion of 44$\mathrm{pmol} / \mu \mathrm{L}$ gramicidin $\mathrm{S}(\mathrm{MW} 1140.7)$ at a mass resolution of approximately 57,000 ( $10 \%$ valley). Experimental conditions were: ESI needle voltage $=8.85 \mathrm{kV} ; \mathrm{HCT}=5.08 \mathrm{kV} ; \mathrm{Sl}=5.02 \mathrm{kV}$; $\mathrm{S} 2=5.00 \mathrm{kV}$; extraction $=4.98 \mathrm{kV}$; focus $1=4.81 \mathrm{kV}$; focus $2=3.22 \mathrm{kV}$; flow rate $5 \mu \mathrm{L} / \mathrm{min}$; filter $1 \mathrm{~ms}$; linear voltage scan; mass range $571.5-573.5$; scan time $=10 \mathrm{~s}$; average of two scans with no smoothing.

gramicidin S is approximately $10-20 \%$ of its signal intensity. At this point, one could achieve higher resolutions $(15,000-20,000)$ for this peak. However, the $14+$ isotope cluster peak of cytochrome $c$ would remain unresolved because of the poor signal-to-noise ratio of the peak. To resolve the isotope cluster of the $14+$ peak of cytochrome $c$, the

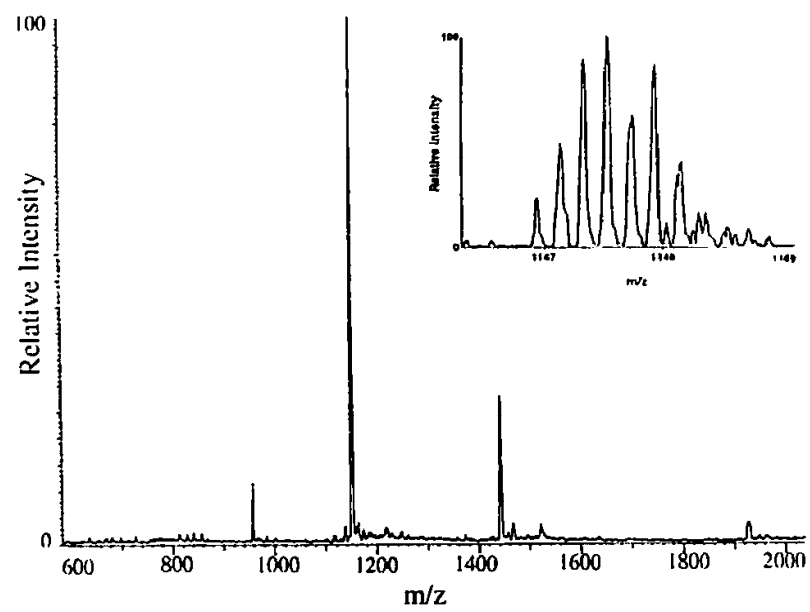

Figure 3. Low resolution $(\sim 1000)$ electrospray mass spectrum of 9-pmol $/ \mu \mathrm{L}$ bovine insulin (MW 5733.6). Experimental conditions were: ESI needle voltage $=8 \mathrm{kV} ; \mathrm{HCT}=5.06 \mathrm{kV} ; \mathrm{S} 1=5.01$ $\mathrm{kV} ; \mathrm{S} 2=4.98 \mathrm{kV}$; extraction $=4.92 \mathrm{kV}$; focus $1=4.78 \mathrm{kV}$; focus $2=3.03 \mathrm{kV}$; flow rate $5 \mu \mathrm{L} / \mathrm{min}$; filter $30 \mathrm{~ms}$; magnet scan; mass range 600-2100; scan time $=20 \mathrm{~s} /$ decade; average of two scans with no smoothing. Inset: High resolution $(\sim 12,000)$ electrospray mass spectrum of $5+$ peak of 9 -pmol $/ \mu \mathrm{L}$ bovine insulin. Experimental conditions were: ESI needle voltage $=7.79 \mathrm{kV}$; HCT $=$ $5.06 \mathrm{kV}$; S1 $=5.01 \mathrm{kV}$; S2 $=4.98 \mathrm{kV}$; extraction $=4.92 \mathrm{kV}$; focus $1=4.78 \mathrm{kV}$; focus $2=3.03 \mathrm{kV}$; flow rate $1 \mu \mathrm{L} / \mathrm{min}$; filter $30 \mathrm{~ms}$; linear voltage scan; mass range 1146-1149; scan time $=20 \mathrm{~s}$; single scan with five points smoothing.

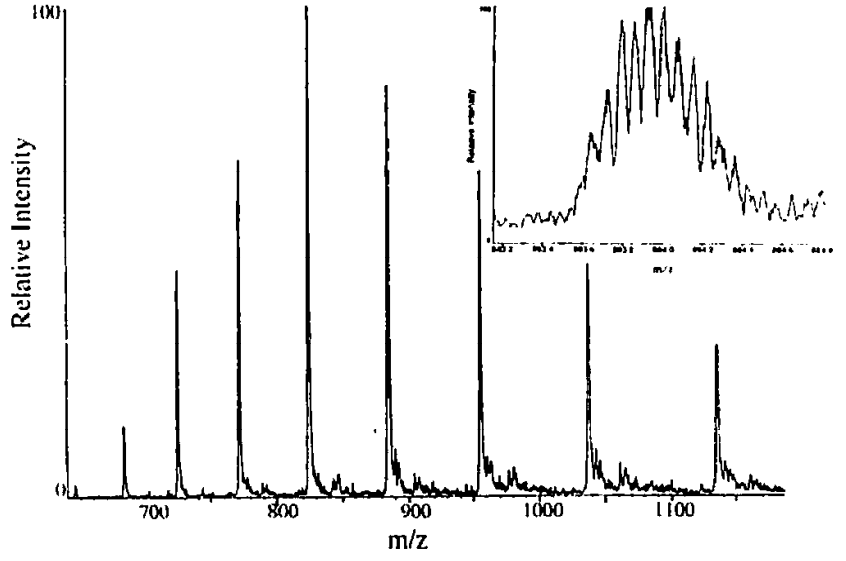

Figure 4. Low resolution ( -1000$)$ electrospray mass spectrum of 50-pmol/ $\mu \mathrm{L}$ cytochrome $c$ (MW 12,360). Experimental conditions were: $\mathrm{ESI}$ needle voltage $=8 \mathrm{kV} ; \mathrm{HCT}=5.09 \mathrm{kV} ; \mathrm{S} 1=5.02$ $\mathrm{kV}$; $\mathrm{S} 2=4.98 \mathrm{kV}$; extraction $=4.94 \mathrm{kV}$; focus $1=4.77 \mathrm{kV}$; focus $2=3.26 \mathrm{kV}$; flow rate $5 \mu \mathrm{L} / \mathrm{min}$; filter $3 \mathrm{~ms}$; magnet scan; mass range $650-1200$; scan time $=20 \mathrm{~s} /$ decade; average of 13 scans with no smoothing. Inset: High resolution $(10,000)$ electrospray mass spectrum of $14+$ peak of $50-\mathrm{pmol} / \mu \mathrm{L}$ cytochrome $c$. Experimental conditions were: $E S I$ needle voltage $=7.48 \mathrm{kV}$; HCT $=5.09 \mathrm{kV} ; \mathrm{S} 1=5.02 \mathrm{kV} ; \mathrm{S} 2=4.98 \mathrm{kV}$; extraction $=4.94 \mathrm{kV}$; focus $1=4.77 \mathrm{kV}$; focus $2=3.26 \mathrm{kV}$; flow rate $3 \mathrm{~mL} / \mathrm{min}$; filter 3 $\mathrm{ms}$; linear voltage scan; mass range $880-884$; scan time $=10 \mathrm{~s}$; average of 100 scans with 11 points smoothing.

signal-to-noise ratio had to be improved by reducing the noise. Noise reduction was achieved by a decrease in the electrospray voltage and then an increase in the distance between the electrospray needle and the HCT until a sudden noise reduction was observed. The signal-to-noise ratio was further improved by a reduction in the flow rates from 3-4 to $1-2 \mu \mathrm{L} / \mathrm{min}$. At this point the distance between the needle and the HCT was approximately $1.5 \mathrm{~cm}$ and the noise level on the doubly charged peak of gramicidin S was approximately 3-5\% of the signal intensity at resolution of approximately 10,000 . The instrument was then tuned to the desired resolution. After tuning by this method, all analyses were performed without retuning the ion optics, and without any changes to the electrospray needle voltage, distance between the electrospray needle and the HCT, or the flow rate.

Under the stated operating conditions, the measured isotope pattern of the doubly charged ion peak of gramicidin $S$ at a mass resolution of approximately 50,000 is as shown in Figure 2. Once high resolution was achieved with gramicidin $\mathrm{S}$, high resolution mass spectrometry of other compounds could be achieved without any adjustment or retuning. However, this was true only when the source and analyzer pressures were low. Under low pressure conditions, the overall performance of our electrospray ionization source is as shown in Figures 3 and 4. As shown, unit mass resolu- 
tions have been achieved for biomolecules with $\mathrm{MW}>$ 12,000 . Accuracy in mass measurement of biological molecules with this design was recently tested by measurement of the average molecular weight of bovine insulin [17]. The average molecular weight of the bovine insulin was obtained by accurate measurement of the centroid of the unresolved isotope cluster of the $[\mathrm{M}+4 \mathrm{H}]^{4+}$ ion at a resolution of approximately 1800. A voltage scan was carried out over a narrow mass range that included a pair of reference peaks (Ultramark 1621) as an interval mass reference standard. The mass range was scanned linearly at a rate of $100 \mathrm{~s} / \mathrm{scan}$. The data were collected in the multichannel analysis mode and were then centroided by an OPUS data system. The measured mass error for the average molecular weight was $1.4 \mathrm{ppm}$.

At higher pressures (such as when a 1-mm skimmer is used for S1 or S2), high resolution was achievable for the doubly charged ion of gramicidin S; however, the isotope clusters of ions with more than two charges, such as the $4+$ ion of bovine insulin (MW 5733.6) and the $14+$ ion of cytochrome $c$ (MW 12,360) remained unresolved. This is probably because of the poor transmission efficiency and/or high kinetic energy spread of these ions at higher pressures due to scattering.

\section{Acknowledgments}

We wish to thank Dr. Barbara Larsen for her helpful discussion during the first stages of this project.

\section{References}

1. Fenn, J. B.; Mann, M.; Meng, C. K.; Wong, S. F.; Whitehouse, C. M. Science 1989, 246, 64-71.'

2. Smith, R. D.; Law, J. A.; Edmonds, C. G.; Barinaga, C. J.; Udseth, H. R. Anal. Chem. 1990, 62, 882-899.

3. Huang, E. C.; Wachs, T.; Conboy, J. J.; Henion, J. D. Anal. Chem. 1990, 62, 713A-725A.

4. Henry, K. D.; Williams, E. R.; Wang, B. H.; McLafferty, F. W.; Shabanowitz, J.; Hunt, D. F. Proc. Natl. Acad. Sci. USA 1989, 86, 9075-9078.

5. Meng, C.; McEwen, C. N.; Larsen, B. S. Rapid Commun. Mass Spectrom. 1990, 4, 147-150; Larsen, B. S.; McEwen, C. N. J. Am. Soc. Mass Spectrom. 1991, 2, 205.

6. Allen, M. H.; Lewis, I. A. S. Rapid Commun. Mass Spectrom. 1989, 3, 255.

7. Van Berkel, G. J.; Glish, G. L.; Mcluckey, S. A. Annl. Chem. 1990, 62, 1284-1295.

8. Schwartz, J. C.; Syka, J. E. P.; Jardine, I. J. Am. Soc. Mass Spectrom. 1990, 2, 198.

9. Qian, M. G.; Lubman, D. M. Anal. Chem. 1995, 67, 234 A.

10. Chapman, J.; Gallagher, R. T.; Barton, E. C.; Curtis, J. M.; Derrick, P. J. Organic Mass Spectrom. 1992, 27, 195-203.

11. Fenn, J. B.; Mann, M.; Meng, C. K.; Wong, S. F.; Whitehouse, C. M. Mass Spectrom. Rev. 1990, 9, 37-70.

12. Chowdhury, S. K.; Katta, V.; Chait, B. T. Rapid Commun. Mass Spectrom. 1990, 4, 81.

13. Jackett, S.; Moini, M. Rev. Sci. Instrum. 1994, 65, 591-596.

14. Wada, Y.; Tamura, J.; Musselman, B. D.; Kassel, D. B.; Sakurai, T.; Matsuo, T. Rapid Commun. Mass Spectrom. 1992, 6, 9.

15. Dobberstein, P.; Schroeder, E. Rapid Commun. Mass Spectrom. 1993, 7, 861-864.

16. Cody, R. B.; Tamura, J.; Musselman, B. Anal. Chemt. 1992, 64, 1561-1570.

17. Moini, M. Rapid Commun. Mass Spectrom. 1994, 8, 711-714. 